\title{
ARTÍCULO
}

\section{La discapacidad intelectual y la discapacidad psicosocial como situaciones de vulnerabilidad ${ }^{\bullet}$}

\section{Intellectual and Psychosocial Disability as Situations of Vulnerability}

\author{
María del Carmen Barraco Avilés \\ Instituto de Derechos Humanos "Gregorio Peces-Barba" \\ Departamento de Derecho Internacional Público, Eclesiástico y Filosofía del Derecho \\ Universidad Carlos III de Madrid \\ https://orcid.org/0000-0001-5027-3750
}

Fecha de recepción 11/06/2019 | De aceptación: 08/10/2021 | De publicación: 23/12/2021

\section{RESUMEN.}

La garantía de los derechos de las personas con discapacidad intelectual y psicosocial pone a prueba a la teoría de los derechos y al modelo social de la discapacidad. En el trabajo se mantiene que el análisis de la discapacidad desde la teoría de la opresión continúa siendo adecuado siempre que se recupere la importancia de la dimensión individual de la discapacidad y se incorpore la representación de la condición humana que deriva de una concepción contemporánea de los derechos y que a su vez es el resultado de la revisión del proyecto de la modernidad desde las teorías críticas.

\section{PALABRAS CLAVE.}

Derechos de las personas con discapacidad; discriminación; capacidad humana; derechos humanos y vulnerabilidad; modelo social de la discapacidad.

\section{ABSTRACT.}

The guarantee of the rights of persons with intellectual and psychosocial disabilities challenges both, the theory of the human rights and the social model of disability. The paper claims that nonetheless, the analysis of the disability from the theory of oppression is still adequate if it attributes the importance it deserves to the individual dimension and incorporates the representation of the human condition derived from a contemporary conception of rights that results from the revision of the modernity project from the critical theories.

\section{KEY WORDS.}

Rights of the persons with disabilities; discrimination; human capacity; human rigths and vulnerability; social model of disability.

\footnotetext{
- Una versión previa de este trabajo ha sido publicada en italiano en la Rivista di filosofía del diritto, 2/2018, pp. 301-320. La revisión y actualización se ha llevado a cabo en el marco del proyecto PID2019-108918GB-I00 financiado por MCIN/EAI/10.13039/501100011033.
} 
Sumario: 1. De qué hablamos cuando hablamos de discapacidad intelectual y psicosocial 1.1. Concepciones de la discapacidad 1.2. La 'discapacidad mental e intelectual' 2. La discapacidad como situación de vulnerabilidad y la vulnerabilidad como opresión 3. Discapacidad intelectual y psicosocial y agencia moral. 4. Sobre los retos de la discapacidad intelectual y psicosocial a las teorías de los derechos. 5. Bibliografía.

\section{De qué hablamos cuando hablamos de discapacidad intelectual y psicosocial}

En la medida en que la definición de la discapacidad y la posibilidad de establecer una tipología de discapacidades están condicionadas por la concepción que está detrás, y que esta a su vez depende de toda una representación de lo que significa ser humano (Oliver 1990,1-11), resulta especialmente ardua la tarea de aclarar qué se quiere decir con 'discapacidad intelectual' y con 'discapacidad psicosocial'. Como es sabido, la Convención Internacional sobre los Derechos de las Personas con Discapacidad (2006) en lugar de ofrecer una definición de discapacidad se refiere a personas con discapacidad como "aquellas que tengan deficiencias físicas, mentales, intelectuales o sensoriales a largo plazo que, al interactuar con diversas barreras, puedan impedir su participación plena y efectiva en la sociedad, en igualdad de condiciones con las demás" (artículo 1.2) ${ }^{1}$.

La Convención incluye a todas las personas con discapacidad, cualquiera que sea la condición individual que, asociada a barreras, impida su inclusión en condiciones de igualdad. El esquema que en mayor medida permite entender esta toma de postura es el modelo social. Con su incorporación, en el Derecho internacional de los derechos humanos se opta por una concepción de la discapacidad que no coincide con lo que de forma predominante se entiende por discapacidad en las sociedades occidentales. Por otro lado, desde el punto de vista de la reflexión teórica, pero con implicaciones en la acción política en favor de los derechos de las personas con discapacidad, es posible identificar variaciones a partir del modelo social y, finalmente, no se trata de una opción indiscutida incluso entre quienes rechazan el modelo médico y rehabilitador que la Convención intenta superar.

En las líneas que siguen se realizará una presentación de estos modelos que se considera necesaria para entender en qué sentido se habla en el trabajo de discapacidad intelectual y psicosocial.

\footnotetext{
${ }^{1}$ El término impairment ha sido traducido al español como 'deficiencia'. Las connotaciones negativas me llevan a mantener el término en inglés o a hablar de 'condición individual'.
} 


\subsection{Concepciones de la discapacidad}

Lamentablemente, todavía hoy en el Derecho y en la concepción social de la discapacidad en diversos lugares del mundo es posible encontrar elementos que encajarían en el modelo, en las antípodas de los derechos humanos, en el que las personas con discapacidad se consideran prescindibles; se habla, en este sentido, de modelo de la prescindencia ${ }^{2}$. En este marco, la discapacidad tiende a considerarse el castigo por acciones pasadas de la persona con discapacidad o sus progenitores y las personas con discapacidad una carga de la que es mejor deshacerse mediante la marginación y, cuando todavía es posible, la adopción de medidas eugenésicas.

Durante amplios períodos en la historia, y también hoy en algunos lugares del mundo y sobre todo en relación con algunos temas, el modelo de tratamiento que predominó fue, precisamente, este enfoque. Se pretendió afrontar la situación mediante la eliminación o la separación de las personas con discapacidad desde presupuestos cuya persistencia en el actual imaginario colectivo se pone de manifiesto a través de figuras como la esterilización, el aborto selectivo, la 'eugenesia', la ocultación, la reclusión o el internamiento involuntario. Los ejemplos citados revelan que desde esta perspectiva se justifican graves vulneraciones de los derechos de las personas con discapacidad.

Puesto que sus presupuestos resultan coherentes con la institucionalización de los Estados de bienestar y con el sentimiento de deuda hacia las numerosas personas que habían resultado heridas en las dos grandes guerras (Barnes, 2012), el modelo que en mayor medida estaba presente en el ámbito occidental en el momento en el que fue aprobada la Convención es el modelo médico o rehabilitador. La discapacidad se considera una carencia individual que se identifica y se aborda con criterios médicos y que supone una desviación del estándar de funcionamiento de un ser humano, por tanto, una 'anormalidad'. Precisamente por la relación entre la discapacidad y la condición del individuo, esta concepción también se caracteriza como individualista, frente al modelo social (Borsay, 1997). Desde este esquema, el tratamiento jurídico político de la discapacidad tiene un carácter tecnocrático, de modo que las intervenciones se justifican con argumentos utilitaristas y tienen como objetivo recuperar a las personas mediante la rehabilitación o evitar el mal mayor que la no intervención supondría también para la sociedad. El fundamento de las medidas que se implementan no es, por tanto, la protección de los derechos de las personas a las que se

\footnotetext{
${ }^{2}$ Se utiliza la tipología y las denominaciones de Agustina Palacios (Palacios, 2008, 37-101).
} 
dirigen, sino mejorar el bienestar global de la sociedad sobre la que se interviene. De algún modo, se trata de convertir en 'normales' a las personas con discapacidad.

El modelo social que, como se apuntaba, está en mayor medida presente en la Convención, supone un cambio de paradigma en el tratamiento de la discapacidad, pero también en relación con la teoría de los derechos humanos (Asís Roig, 2013). En este marco, la discapacidad se relaciona con la discriminación, y, en esta medida, puede considerarse como una cuestión relevante desde el punto de vista de los derechos. El modelo se articula por contraposición a los planteamientos del modelo médico y entre sus presupuestos se subraya que el modo en el que una característica individual influye en la capacidad de funcionar de las personas tiene que ver con el entorno, de forma que, en definitiva, la definición de la normalidad es una cuestión de poder. En el origen del modelo se encuentra la acción política de las propias personas con discapacidad, que termina dando pie al desarrollo en la academia de los Disability Studies.

Frente al modelo de la prescindencia, que niega el valor de las personas con discapacidad, y al modelo rehabilitador, que trata de adecuarlas a la normalidad, el modelo social sería más coherente con la afirmación del igual valor de todos los seres humanos y, en esta medida, con abordar la discapacidad desde políticas públicas basadas en derechos humanos (Quinn and Degener, 2002) en las que el objetivo son los derechos, el método la participación y el resultado el empoderamiento de las personas en cuyo favor se interviene.

El modelo social se articula de forma diferente en los Estados Unidos y en Reino Unido. Así, en el esquema estadounidense, donde tiene un gran peso el movimiento de vida independiente, la discapacidad se considera una manifestación de la diversidad humana y el análisis de la situación de las personas con discapacidad se realiza en el marco de la discusión sobre los derechos de las minorías (Shapiro, 1994). Si bien es cierto que las enseñanzas de este movimiento americano en relación con la relevancia de la vida independiente han sido muy importantes también desde el punto de vista de la discapacidad intelectual y psicosocial, algunos de los planteamientos, especialmente en el plano académico, mantienen una concepción liberal de los derechos, con lo que, entre otros problemas, el concepto de discriminación que se maneja tiende a desconsiderar las dimensiones estructurales y se conserva una imagen del titular de derechos que no tiene en cuenta que la vulnerabilidad es un rasgo característico de la condición 
humana. Sobre esta cuestión, como veremos, se insiste en posteriores revisiones y críticas del modelo social de la discapacidad en su conjunto.

A diferencia de la versión americana, la construcción del modelo social predominante en el contexto británico insiste en que la discapacidad es el resultado de estructuras sociales de dominación. De este modo, y con una gran influencia del materialismo histórico (Meekosha and Shuttleworth, 2009, 50), la discapacidad se representa como una situación de opresión; son las estructuras económicas, sociales y políticas las que apuntalan el sistema de valores que estigmatiza a las personas con discapacidad (Borsay, 1997). T. Shakespeare (Shakespeare and Watson, 2002) atribuye a Vic Finkelstein (Filkenstein 1980 y 1981), Colin Barnes (Barnes, 1991) y Mike Oliver (Oliver, 1990) el mérito de dotar de 'credibilidad académica' a un modelo surgido del movimiento asociativo.

A partir de un cierto momento, no sin críticas y precauciones, también se produjo una alineación del modelo social británico con el enfoque basado en derechos. Entre las críticas, por ejemplo, en un documento de balance sobre el movimiento en Gran Bretaña, Barnes y Oliver apuntan que la focalización en la idea de la discapacidad como una cuestión de derechos no resuelve el problema de discriminación y opresión por razón de discapacidad (Oliver and Barnes, 2006). Más crítico se muestra Finkelstein, quien, citando precisamente como referencia a Oliver, sitúa esta complicidad entre el modelo social británico de la discapacidad y los derechos humanos en los años 90. En opinión de Finkelstein, el enfoque en derechos presenta dos problemas relacionados. Señala el autor que, en la medida en que el discurso de los derechos se centra en las características del individuo, también es fácil desde él desconsiderar que la situación de las personas con discapacidad es el resultado del modo en que la sociedad está organizada y estructurada, con lo que, afirma, la identificación del modelo social con los derechos "encaja perfectamente con el programa de privatización del nuevo laborismo para un libre mercado de salud y servicios sociales" (Finkestein, 2007).

Las críticas a esta versión del modelo social considerada rígida, que representan tanto Finkelstein como Barnes y Oliver, proceden de diversos frentes. En este momento interesa hacer referencia a dos. Por un lado, se señala que se trata de un esquema desde el que se asume la representación del ser humano que está presente en la concepción tradicional de los derechos, a pesar de que esta representación ha sido un obstáculo para que los derechos llegaran a ser una herramienta adecuada para la salvaguarda de la dignidad de las personas con discapacidad y de todas aquéllas que como ellas son adscritas a los llamados 
'grupos vulnerables'. Adicionalmente, desde distintas perspectivas, se considera que una parte de las limitaciones del modelo tienen que ver con el papel que en él desempeña la dimensión individual de la discapacidad.

Efectivamente, el concepto de autonomía que se incorpora a la defensa de la vida independiente, tanto en la versión americana como en la versión británica del modelo social, puede ser criticado desde una perspectiva relacional. Y es que, en ambos casos, aunque muy claramente en el modelo británico, se rechaza la ideología que define la independencia a partir de la habilidad para realizar tareas sin ayuda, también se sigue incorporando la idea de que la independencia es una característica del titular de derechos, eso sí, definida como control, lo que vincula la ideología del modelo social con un modelo racional-voluntarista de autonomía (Reindal, 1999, 354). Es cierto que esta revisión puede ser adecuada para incluir a las personas cuya racionalidad no se discute, pero la exclusión permanece justificada en el caso de las personas con discapacidad psicosocial e intelectual, dado que precisamente es su racionalidad y con ella la capacidad de control, la que se pone en duda.

Para entender el modo en el que el modelo social sigue siendo excluyente, Siebers pone como ejemplo la reflexión sobre las barreras en relación con el derecho de participación política. A propósito de este derecho resulta evidente que a pesar de que puede haber barreras que dificulten la participación política de las personas cuyo impairment es de carácter físico o sensorial, difícilmente en las democracias occidentales se cuestiona que sean titulares del derecho de voto, a diferencia de lo que ocurre con las personas con discapacidad intelectual que son privadas de este derecho en la mayoría de los contextos democráticos (Siebers, 2008, 78). A título de ejemplo, pueden considerarse los argumentos esgrimidos en nuestro país (Cuenca Gómez, 2018) incluso después de que la Ley Orgánica 2/2018, de 5 de diciembre, para la modificación de la Ley Orgánica 5/1985, de 19 de junio, de Régimen Electoral General para garantizar el derecho de sufragio de todas las personas con discapacidad (BOE núm 296, de 6 de diciembre de 2018) restituyera el derecho de sufragio a las personas que habían sido privadas de él (Barranco Avilés, M.C., 2019).

A propósito de las críticas al modelo social que toman como punto de referencia el papel que desempeña la condición personal en la caracterización de la discapacidad, Tom Shakespeare y Nicholas Watson se refieren a tres aspectos. En primer lugar, y a la inversa de las objeciones de Finkelstein, se ha considerado que el situar el foco de la intervención casi exclusivamente en las estructuras sociales discapacitantes 
impide ver la diferencia entre la discriminación que viven las personas con discapacidad y la que viven otras personas, como las mujeres. Como consecuencia de esta reflexión, algunos teóricos y teóricas proponen recuperar el papel del impairment en la definición de discapacidad, aunque consideran que el modelo social sigue manteniendo su utilidad. Se puede hablar de estas posiciones como modelo social intermedio o moderado (Bernardini, 2016, 50-63). Es necesario advertir que cuando desde ellas se propone revisar la perspectiva para incluir la dimensión individual, de ningún modo se trata de construirla desde un punto de vista exclusivamente biológico y médico, sino, por el contrario, el objetivo es reivindicar que no es posible tener una idea clara de qué significa la discapacidad y, por tanto, que no es posible revertir la opresión que sufren las personas con discapacidad, si no se tiene en cuenta que se trata de una situación en la que la dimensión individual condiciona el modo en el que el sistema de opresión opera, al tiempo que el sistema de opresión condiciona el modo en el que es entendida la misma condición individual.

Frente a estas posiciones que se mantienen en el modelo social, aunque revisado, autores como Shakespeare y Watson consideran que el modelo social es un paradigma superado. En su opinión, la desconsideración de la condición individual de las personas con discapacidad en el marco de los Disability Studies resulta tan problemática como lo fue para el feminismo prescindir de la referencia a las mujeres. Los autores citados, se alinean con ello con los Feminist Disability Studies en la idea de que el impairment es parte de la experiencia diaria de las personas con discapacidad y de que precisamente por ello debe ser tenido en cuenta, tanto en la teoría social, como en las estrategias políticas. De este modo, y en lo que a los asuntos de mayor interés para este trabajo se refiere, frente a la excesiva fragmentación de la discapacidad en el modelo médico y la homogenización en el modelo social, el objetivo de rescatar el peso de la dimensión individual es poner de manifiesto que distintos grupos de impairments pueden tener implicaciones diferentes (Shakespeare and Watson, 2002). El modelo social es rechazado en parte porque, llevado a su extremo, permite afirmar que en ningún caso el impairment es algo que deba evitarse o reducirse, si es posible hacerlo. Conviene tener en cuenta que la reflexión no supone una valoración negativa de las personas con discapacidad o que sus vidas se consideren de menor valor, se trata de subrayar que el modelo social ha dejado de ser útil precisamente porque en sus discursos la condición personal está ausente.

Desde el punto de vista teórico, estas propuestas críticas del modelo social consideran que el descuido de la condición individual de las personas con discapacidad se debe, en el caso de los Disability Studies 
británicos, al mantenimiento de la “ortodoxia del neo-marxismo, del materialismo histórico y del modelo social" (Corker and Shakespeare, 2002, 13). Recogiendo algunas piezas del postmodernismo ${ }^{3}$, desde esta posición se cuestiona la dicotomía entre e discapacidad e impairment, del mismo modo que el feminismo postmoderno cuestiona la dicotomía entre el género y el sexo. No obstante, ni el feminismo puede prescindir de la idea de mujeres (aunque sea una idea provisional y aunque se reconozca que la representación de las mujeres está tamizada por elementos culturales y de poder, que el sexo no es meramente biología y que los sexos no son sólo dos), ni el discurso de la discapacidad puede construirse de espaldas a la representación del impairment. En palabras de Siebers, "la fisicalidad es parte de la realidad del cuerpo discapacitado, y si la corporeización contribuye a la experiencia de las personas con discapacidades, su tergiversación como una condición mental tendrá un efecto perjudicial en su capacidad para organizarse políticamente" (Siebers, 2008, 77).

Finalmente, Shakespeare y Watson critican la exigencia, que consideran parte del modelo social, de que las personas con discapacidad se identifiquen políticamente como tales. En primer lugar, porque algunas personas con discapacidad rechazan esta identidad, pero también porque las identidades son complejas, de modo que las personas con discapacidad no son sólo personas con discapacidad. Esta y las anteriores reflexiones sirven de argumento a los autores para proponer que ha llegado el momento de sustituir el modelo social por un nuevo paradigma que tenga en cuenta, entre otras ideas, que existe una línea de continuidad entre impairment y discapacidad; que la discapacidad no puede ser reducida a una cuestión de barreras sociales; y que se debe rechazar que la identidad de la persona con discapacidad está dada. El último matiz resulta especialmente interesante desde la perspectiva del presente trabajo porque pone de manifiesto que el modelo social asume una concepción del sujeto del modelo tradicional de derechos en su representación de la opresión de las personas con discapacidad, de modo que no cuestiona el presupuesto de la ideología capacitista conforme al cual algunos seres humanos no son vulnerables. Frente a ello, Shakespeare y Watson instan a romper la línea entre personas con y sin discapacidad desde la constatación de que todo el mundo es 'impaired' (Shakespeare and Watson, 2002).

La propuesta resulta útil como un ariete para la deconstrucción de la imagen del ser humano subyacente al discurso tradicional de los derechos. Efectivamente, las críticas contextualistas a las teorías de los

\footnotetext{
${ }^{3}$ Sobre la relación entre la teoría queer y los Disability Studies, puede verse McRuer, 2021 y la entrevista al autor publicada por Moscoso Pérez y Arnau Ripollés (2016).
} 
derechos ponen de manifiesto, por ejemplo, que la abstracción inhabilita a los derechos en relación con el sufrimiento humano. Además, difícilmente desde un esquema en el que los rasgos definitorios de la agencia moral son la racionalidad y la capacidad de autodeterminación es posible justificar la igual dignidad de todos los seres humanos. Estas deficiencias de partida del modelo tradicional de derechos resultan especialmente excluyentes en relación con aquellos sujetos que, como las personas con discapacidad intelectual o psicosocial, no son considerados racionales. Es interesante recordar, por otro lado, que también los niños y niñas quedan fuera de la representación del titular de derechos y que en tiempos lo fueron las mujeres y los trabajadores asalariados (Kant, 1793).

Frente a estas propuestas de revisión, desde algunas posiciones se considera que las críticas postmodernas al modelo social se sitúan en la tendencia general de 'desradicalización de las ciencias sociales' que acompaña la desintegración del bloque comunista y consideran que, más allá de disquisiciones teóricas, el modelo social continúa siendo el más útil para la revisión de las instituciones en un contexto en el que las personas con discapacidad siguen siendo 'las más pobres de entre las pobres' (Barnes, 2012). Si bien es importante atender a esta advertencia, también es cierto que sólo desde la incorporación de una concepción revisada de lo que implica ser humano o humana al modelo -que supone, por otra parte, prestar atención a los sujetos y no sólo a la sociedad- es posible revertir la opresión de las personas con discapacidad intelectual y psicosocial. De este modo, la tesis que se mantiene en el presente trabajo es que la vía más provechosa para la defensa de los derechos de las personas con discapacidad intelectual es la que, en la línea de los Critical Disability Studies (Meekosha and Shuttleworth, 2009), tiene en cuenta que existe una 'relación mutuamente constitutiva entre impairment and disability', que es tanto material como discursivamente producida (Corker and French 1999, 6). Desde esta caracterización se abordará la explicación de qué significan la discapacidad mental y la discapacidad intelectual, se presentará un esbozo del modo en el que opera la opresión sobre las personas con discapacidad intelectual y psicosocial y se abordará el problema de la capacidad que es, en mi opinión, el aspecto más relevante en la representación de la condición individual de las personas con discapacidad intelectual y psicosocial.

\subsection{La 'discapacidad mental e intelectual'}

Como se ha apuntado, la Convención se refiere a impairments de carácter físico, mental, intelectual o sensorial. Con este punto de partida, es posible afirmar que el objeto de este trabajo tiene que ver con las personas cuya discapacidad resulta de la interacción entre sus condiciones mentales y/o intelectuales y 
las barreras. También se ha apuntado que la representación de esas condiciones en nuestro contexto cultural aleja a estas personas de la concepción ideal del ser humano que está detrás de los sistemas de protección de los derechos, con lo que, incluso en los contextos democráticos, se justifican instituciones que sólo encuentran explicación en el modelo de la prescindencia.

Se prefiere la denominación de discapacidad psicosocial para hacer referencia a la discapacidad que la Convención parece asociar con condiciones mentales, porque la denominación de discapacidad mental se ha utilizado para hacer referencia también a la que resulta de las condiciones intelectuales. No obstante, no se pretende cerrar la discusión sobre la denominación de estas situaciones, que también permanece abierta en los estudios sobre la discapacidad. El objetivo aquí es solamente hacer una propuesta que sea útil para entender en qué medida la discapacidad intelectual y psicosocial pone a prueba a los derechos como herramienta de salvaguarda de la dignidad de un modo distinto a como lo hace la discapacidad física y sensorial.

Las personas con discapacidad intelectual y psicosocial son las que se alejan en mayor medida de los rasgos que habitualmente se asocian a la dignidad y de los que se hace depender la atribución de derechos. Efectivamente, las personas con discapacidad intelectual y psicosocial se consideran dependientes y no autosuficientes (por tanto, no autónomas) y, lo que influye tal vez en mayor medida, desprovistas de razón. Este carácter 'irracional' con el que se dibuja a las personas con discapacidad intelectual y psicosocial las convierte en eternos niños y niñas o en seres peligrosos, respectivamente. La imagen que se tiene de las personas con discapacidad intelectual y psicosocial, unida a los rasgos que en un modelo tradicional de derechos se consideran definitorios de la dignidad humana generan grandes dificultades para la implementación de la Convención, que encaja mejor con la representación de la discapacidad física y sensorial.

Desde una definición que puede resultar coherente con el modelo social, podemos decir que la forma de aprender, comprender y comunicarse de las personas, al interactuar con el modo en el que se organiza el aprendizaje, la comprensión y la comunicación en la sociedad genera una discapacidad intelectual.

En consecuencia, la aproximación a la discapacidad intelectual desde el modelo social implica ser capaces de identificar las barreras que impiden que las personas que aprenden comprenden y se comunican de un cierto modo puedan ser incluidas en la comunidad. Un punto importante al respecto es que no hay un único tipo o manifestación de la discapacidad intelectual. Las personas consideradas con 
discapacidad intelectual son muy diversas entre sí. La actitud que socialmente y, a partir de ahí, jurídicamente, se suele adoptar en relación con las personas con discapacidad intelectual es el paternalismo, lo que impide que puedan realizarse y participar en condiciones de igualdad con el resto de las personas. Precisamente porque se considera que no pueden cuidarse por sí mismas, las personas con discapacidad viven siempre sometidas al poder de otras personas.

Por su parte, puede decirse que la discapacidad psicosocial es "un producto social que resulta de la interacción entre una persona con un 'proceso psico-afectivo' y las barreras actitudinales y del entorno que la sociedad genera; y que, teniendo como base el estigma, el miedo y la ignorancia, limitan su participación plena en igualdad de condiciones con las demás” (Poder Judicial de Costa Rica, 2013, 23)4. En definitiva, las personas con discapacidad psicosocial son personas cuya forma de interactuar no es aceptada socialmente. A las personas con discapacidad psicosocial se las margina y se las teme, de modo que las barreras actitudinales hacen que en la mayor parte de las sociedades las personas con discapacidad psicosocial se vean insertas en un esquema de prescindencia. La denominación de discapacidad psicosocial por la que aquí se opta, implica dejar de lado el modelo médico, sin embargo, las instituciones todavía permanecen ancladas en el modelo médico y en la perspectiva biologicista de la salud mental.

Aunque la medicalización de la discapacidad psicosocial es difícilmente compatible con el modelo social de la discapacidad, la reflexión desde la bioética ha acercado el tratamiento de la salud mental a los principios de los derechos humanos y ha propiciado su abordaje desde un planteamiento holístico, de modo que parece posible una aproximación coherente con la Convención también en el ámbito médico, aunque, sin embargo, esta no es la predominante.

En este sentido, es de interés recordar que el Informe del Relator Especial sobre el derecho de toda persona al disfrute del más alto nivel posible de salud física y mental de 2017 (A / señalaba varios problemas del tratamiento contemporáneo de la salud mental. En primer lugar, se refería precisamente al predominio del modelo biomédico frente al modelo psicosocial que "entiende las experiencias psicológicas y sociales como factores de riesgo que contribuyen a una mala salud mental y como factores positivos que contribuyen al bienestar", en el que las intervenciones se orientan a

\footnotetext{
${ }^{4}$ Incorpora la definición de OEA, Declaración de Antigua sobre el ejercicio de la Capacidad Jurídica de las Personas con Discapacidad Psicosocial en América Latina, 43 Asamblea General de la OEA, "Foro Latinoamericano sobre capacidad legal de las personas con discapacidad: Sin capacidad jurídica, no hay derechos humanos”, La Antigua Guatemala, 5 de junio de 2013.
} 
empoderar a los destinatarios y que se aleja "de la suposición arbitraria de que las intervenciones biomédicas son más eficaces” (\$20). Asimismo, el Relator pone de manifiesto las asimetrías de poder que se producen en el marco del modelo biomédico, inspirado en el paternalismo e imbuido de la idea de que las personas con discapacidad social son peligrosas e incompetentes para tomar decisiones por sí mismas. En el informe se subraya que las asimetrías se han visto agravadas por el poder financiero de la industria farmacéutica. En el informe de 2020 se denuncia la inacción mundial "para hacer frente a las vulneraciones de los derechos humanos en los sistemas de atención a la salud mental” (abril 2020 $\underline{\mathrm{A} / \mathrm{HRC} / 44 / 48,}, \S 82)$.

En relación con la discapacidad intelectual y con la discapacidad psicosocial, se evidencia que las teorías tradicionales de los derechos y el modelo social rígido de la discapacidad se han construido sobre presupuestos que dejan fuera del proyecto emancipador a algunas personas. El principal problema de una y otra aproximación tiene que ver con el ideal de ser humano invulnerable sobre el que se construyen, en el que no hay sitio para quienes se considera que no pueden ser racionales (Carlson, 2009). Frente a estas aproximaciones, en los siguientes apartados se abordará la discapacidad intelectual y psicosocial desde una teoría contemporánea de derechos y un modelo social revisado de la discapacidad.

\section{La dimensión social: la discapacidad como situación de vulnerabilidad y la vulnerabilidad como} opresión

La alianza entre el modelo social de la discapacidad y la teoría de los derechos ha resultado especialmente interesante con respecto a la consideración de la vulnerabilidad en su relación con los derechos humanos. Efectivamente, frente a una situación en la que la referencia a grupos vulnerables implica que hay ciertos sujetos que comparten una debilidad personal que los aleja de la normalidad y que los hace acreedores de especial protección y cuidado, la influencia del modelo social permite entender la vulnerabilidad como una situación de opresión (Barranco Avilés 2014). De este modo, un grupo vulnerable es el conformado por personas que en puntos importantes se encuentran en una posición de desventaja, precisamente debido a la cualidad que los identifica como miembros de ese grupo; además, la posición de desventaja no es natural ni inevitable, sino que está ideológicamente justificada y ‘alguien' obtiene beneficios de ella (Abberley, 1987, 7).

En este esquema, la discapacidad no guarda relación exclusiva con las características de las personas, sino que, por un lado, puede verse como resultado de la estructura social que genera dominación - 
obstáculos a la autonomía- y opresión -obstáculos a la autorrealización- (Young, 2011, 39-65) y, por otro, las características de las personas se conceptualizan a partir de esas mismas estructuras. La raza, el sexo y la discapacidad tienen en común, como situaciones de vulnerabilidad, que en relación con ellos se han construido estereotipos que generan opresión.

Las personas que no se ajustan al estándar encuentran dificultades para ser incluidas, porque la sociedad está construida desde los parámetros de la 'normalidad'. Es importante tener en cuenta que las estructuras que vulnerabilizan no son universales, sino contextuales, de modo que no se puede hablar de grupos 'universalmente' vulnerables. Los grupos de personas en situación de vulnerabilidad son diferentes en los diferentes contextos y, en ocasiones, lo que convierte a estas personas en grupo es precisamente que son víctimas en ese contexto de una misma forma de discriminación. Podemos pensar en las mujeres, pero también en las personas con discapacidad en general y, más en concreto, con discapacidad intelectual y con discapacidad psicosocial.

Como ya hiciera Barnes en relación con la discapacidad (Barnes, Mercer and Shakespeare 1999, 80), para entender cómo se manifiesta la opresión en relación con las personas con discapacidad intelectual y psicosocial, es útil el ejercicio de utilizar las cinco caras de la opresión de Young: imperialismo cultural, marginación, privación de poder, explotación y violencia. Comenzando por la primera de estas caras, el imperialismo se revela en el grado en que nuestras sociedades no se consideran valiosas las formas de comprender e interactuar de las personas con discapacidad intelectual y psicosocial.

Por otro lado, las personas con discapacidad intelectual y psicosocial son marginadas y esto está en numerosas ocasiones amparado por normas jurídicas. La marginación para ambas situaciones se manifiesta en la frecuencia con la que estas personas con discapacidad son institucionalizadas; para las personas con discapacidad psicosocial se justifica el internamiento involuntario, que en numerosas ocasiones tiene lugar en centros específicamente habilitados para este fin. Las personas con discapacidad intelectual son segregadas desde la más tierna infancia a través de su derivación frecuente a centros educativos especiales o de su ubicación en espacios separados en centros ordinarios. La crisis financiera (Campoy, 2016 y Troilo, 2012) acentuó estos problemas de segregación en el ámbito escolar y la gestión de la educación durante la pandemia ha generado todavía más barreras para la educación de niños y niñas con discapacidad (UNICEF, 2020, 14). 
Una de las formas en las que de modo más escandaloso se manifiesta la opresión de las personas a las que nos referimos es la privación de poder. Como veremos, esta materia se relaciona directamente con la conceptualización de las personas con discapacidad intelectual y psicosocial como carentes de racionalidad y se manifiesta en instituciones tan arraigadas en nuestros sistemas jurídicos como la sustitución en la toma de decisiones, el tratamiento involuntario y la institucionalización forzada.

La explotación es una situación cotidiana para las personas con discapacidad intelectual. En relación con ellas, se produce la paradoja de que al mismo tiempo que se niega su capacidad para trabajar y, con ello, para ser miembros útiles de la sociedad, se utiliza su trabajo como mano de obra barata y precaria. Conviene no olvidar, por otro lado, que una gran cantidad de personas con discapacidad (sobre todo mujeres) están realizando tarea de cuidado, como se sabe, no reconocidas y escasa o nulamente remuneradas (Hughes 2016) ${ }^{5}$. Al respecto resultan de interés los datos sobre el salario de las personas con discapacidad; en 2018 los resultados muestran que los hombres con discapacidad tuvieron un salario un $15,9 \%$ inferior al de los hombres sin discapacidad, que el salario de las mujeres con discapacidad fue un $14,9 \%$ menor que el de las mujeres sin discapacidad y que los salarios más bajos correspondieron a los trabajadores con discapacidad intelectual y psicosocial (INE, 2020).

En el apartado de la violencia, también es el grupo de personas con discapacidad sobre el que con más frecuencia siguen considerándose legales la esterilización ${ }^{6}$ y la realización de abortos selectivos, a veces incluso forzados. La incidencia de agresiones y abusos es mayor entre las personas con discapacidad intelectual, sobre todo entre las mujeres que también son más frecuentemente víctimas de violencia de género (Sierra, 2018, 412 ss.).

Esta opresión característica a la que se somete a las personas con discapacidad intelectual y psicosocial no puede entenderse si además no se analiza la representación de la condición individual de estas personas.

\footnotetext{
${ }^{5}$ Pueden verse también los datos sobre el salario de las personas con discapacidad del), sobre los datos de 2018, los resultados muestran que los hombres con discapacidad tuvieron un salario un $15,9 \%$ inferior al de los hombres sin discapacidad que el salario de las mujeres con discapacidad fue un $14,9 \%$ menor que el de las mujeres sin discapacidad y que los salarios más bajos correspondieron a los trabajadores con discapacidad intelectual y psicosocial.

${ }^{6}$ En España, la Ley Orgánica 2/2020, de 16 de diciembre erradicó la esterilización forzosa hasta su entrada en vigor amparada por el artículo 156.2 del Código Penal.
} 


\section{Discapacidad intelectual y psicosocial y agencia moral}

Las personas con discapacidad intelectual y psicosocial son representadas como vulnerables en un sentido que choca frontalmente con la conceptualización de la condición humana en la concepción tradicional de los derechos. Son irracionales o débiles de mente. Esta imagen impide que se consideren sujetos de Derecho, lo que implica a su vez que son irrelevantes desde el punto de vista de la democracia y de los derechos. La cuestión es importante porque la condición de las personas con discapacidad intelectual y psicosocial se utiliza como argumento para dejarlos fuera de las garantías que se implementan para que la dignidad humana no se vea afectada por la convivencia con otros sujetos. Además, sólo de forma reciente y en el marco de los Disability Studies la exclusión de las personas con discapacidad intelectual y psicosocial se ha cuestionado (Simplican 2015, 201).

Si bien la mentalidad heredada del humanismo renacentista y de la Ilustración puede justificar fallos en el cuerpo, los fallos en la racionalidad impiden reconocer la igual dignidad de quienes los presentan. La condición biológica aproxima a los seres humanos y a los animales, mientras la razón es precisamente el rasgo distintivo de la especie. Los movimientos de defensa de los derechos humanos que han hecho frente a las distintas exclusiones derivadas del modelo han esgrimido como bandera que los seres humanos excluidos eran capaces de demostrar que comparten la condición humana así entendida (Kittay 2005, 98).

Es desde esta perspectiva desde la que es posible explicar la pervivencia del modelo de la prescincencia y del modelo médico en las prácticas e instituciones actuales, en cuyo marco se lesionan los derechos de las personas con discapacidad intelectual y psicosocial (Ryan and Thomas 1980, 14). La incidencia del modelo social rígido sobre las políticas y las normas de la discapacidad tampoco ha conseguido romper con esta dinámica en la atribución de dignidad y con ella de derechos. En el marco del modelo, la concepción de la autonomía todavía resulta excluyente de las personas con discapacidad intelectual y también con discapacidad psicosocial. En este sentido, dice Simplican, en relación con el modelo social de discapacidad, "incluso cuando pretenden ser inclusivos, los estudiosos de la discapacidad y los activistas de los derechos de la discapacidad a menudo recurren a normas cognitivas de autonomía y empoderamiento que vuelven a trazar los límites de la personalidad y reifican la exclusión” (Simplican, 2015). 
La condición individual de las personas con discapacidad intelectual y psicosocial se representa en buena medida a partir de su imposibilidad de gobernarse por sí mismas. Ya que esta circunstancia se considera un dato de su condición individual, la privación de poder es la cara de la opresión que de un modo más importante se revela en relación con quienes son así categorizadas. En este aspecto, los argumentos que justifican el estatuto jurídico y social de las personas con discapacidad reproducen los argumentos esgrimidos para legitimar la desventaja de las mujeres en épocas pasadas. Como hoy ocurre con las personas con discapacidad intelectual y psicosocial, las mujeres resultaron privadas social, política y jurídicamente de capacidad porque la ausencia de racionalidad formaba parte de la representación de lo que significaba ser mujer. En buena medida, la inclusión de las mujeres ha venido marcada por el grado en que se ha aceptado que el sexo no condiciona esta cualidad. Sin embargo, la reflexión que arranca de la crítica feminista pone de manifiesto también que la priorización de la razón frente a otros rasgos de carácter de los seres humanos, como la emoción (Tronto 1993, 24-59), o frente a la corporalidad, es arbitraria.

Para entender cómo operan los estereotipos que pesan sobre las personas con discapacidad intelectual y psicosocial, resulta útil prestar atención a la medida en que estas personas son consideradas sujetos de derecho y, por tanto, están empoderadas. De este modo, el enfoque se desplaza desde la discriminación a la capacidad tanto en sentido formal, que implica la posibilidad de las personas de ejercer por sí mismas los derechos y deberes, como en sentido material, que implica ser reconocidas socialmente como adultas (Freeman, 1989-1990, 112-113).

En relación con la dimensión formal de la capacidad, el artículo 12 de la Convención sobre los Derechos de las Personas con Discapacidad genera la necesidad de revisar los sistemas occidentales tradicionales, basados en la sustitución en la toma de decisiones de los sujetos considerados incapaces, por sistemas en los que el objetivo de la intervención jurídica es el establecimiento de apoyos en la toma de decisiones. El impacto de esta determinación es enorme, puesto que pone de manifiesto, en primer lugar, que la condición individual es importante para comprender la discapacidad. Esta circunstancia se ratifica si se tiene en cuenta que en las democracias occidentales las resistencias a la implementación de este artículo se producen en relación con las personas con discapacidad intelectual y psicosocial, que es en relación con las que se mantienen los procesos de incapacitación. Nuevamente, el ejemplo lo encontramos en las dificultades en nuestro país (Cuenca Gómez, 2011). 
Por otro lado, el precepto sólo se entiende desde el reconocimiento de que los seres humanos, todos los seres humanos, decidimos respaldados por las personas que nos rodean (Cuenca Gómez, 2012, 130), de modo que además de una representación alternativa de las personas con discapacidad, introduce una representación alternativa de lo que significa ser humano.

Finalmente, el artículo 12 no sólo afecta a las normas que específicamente se refieren a la capacidad jurídica, cuyo ámbito de referencia venían siendo las cuestiones patrimoniales, por tanto, no sólo se refiere a la capacidad formal. Por el contrario, en la medida en que reconoce el derecho al igual reconocimiento como persona ante la ley, su ámbito de aplicación se refiere a todas las circunstancias en las que los sujetos de derecho tomamos decisiones incluyendo desde la disposición sobre el propio cuerpo hasta la posibilidad de participar en política ${ }^{7}$. Esta dimensión material de la capacidad enlaza, en definitiva, con el derecho a tener derechos que implica que las personas son consideradas sujetos y no meros objetos de derecho y depende de su pertenencia a una comunidad política (Siebers, 2008, 176).

La representación de la condición humana desde la concepción tradicional de los derechos, compartida en buena medida por el modelo social rígido de la discapacidad, resulta arbitraria y obedece al ideal que quienes construyen el modelo mantienen a partir de su propia experiencia. En contraposición, la concepción contemporánea de los derechos incorpora una concepción abierta e inclusiva de la dignidad humana que implica la contextualización de la existencia y, con ella, la relevancia del cuerpo, de la vulnerabilidad y de la dependencia como rasgos de la humanidad relevantes para la conceptualización de los derechos.

Por otro lado, y desde el punto de vista de la legitimidad de las instituciones, la exclusión de las personas con discapacidad de los procesos políticos implica un déficit democrático desde una concepción de la democracia, más coherente con esa concepción contemporánea, vinculada a la inclusión y no tanto a la racionalidad y al consenso (Simplican, 2012, 212).

Como se ha podido ver, la discapacidad intelectual y psicosocial ponen a prueba tanto la teoría de los derechos humanos como el modelo social de la discapacidad. Si se tiene en cuenta que de lo que se trata es de discutir la racionalidad como el rasgo distintivo del que deriva la dignidad humana y que el estereotipo desde el que se representan las personas con discapacidad intelectual y psicosocial lleva

\footnotetext{
${ }^{7}$ En relación con la discapacidad psicosocial ver Barnes and Shardlow, 1996.
} 
precisamente a negar la racionalidad, la estrategia seguida por otros movimientos consistente en reconstruir el estereotipo se muestra especialmente inadecuada.

Frente a ello, en relación con la discapacidad intelectual y psicosocial se muestra en mayor medida que la universalidad de los derechos requiere una revisión de los presupuestos desde los que se caracteriza la condición humana como presupuesto de la dignidad (sin prejuzgar el valor de otros seres), así como una recuperación del peso de la condición individual cuando de lo que se trata es de la defensa de los derechos de las personas con discapacidad.

\section{A modo de conclusión: sobre los retos de la discapacidad intelectual y psicosocial a los estudios de la discapacidad y a las teorías de los derechos}

El modelo social de la discapacidad tuvo una gran influencia en la Convención sobre los Derechos de las Personas con Discapacidad y abrió la puerta a la posibilidad de abordar la discapacidad y, en general, la vulnerabilidad, desde un enfoque basado en derechos, sin embargo, ni en su versión americana ni en su versión inglesa puso en cuestión la representación de la condición humana que desde el que se justifica la exclusión de las personas con discapacidad y que se basa en la razón y la autonomía como elementos sobre los que se articula el significado de la humano. La desconsideración de la vulnerabilidad como una condición humana universal y la atención casi exclusiva a las barreras como componente de la discapacidad, hacen perder de vista la dimensión individual de una y otra y dificulta la reflexión sobre las garantías adecuadas a implementar para que los derechos sean verdaderamente universales. Y estos problemas se ponen de manifiesto si se considera con detenimiento la situación de las personas con discapacidad intelectual y psicosocial.

Efectivamente, las discusiones desarrolladas en torno a cuestiones tales como el reconocimiento del derecho de voto o la justificación de la incapacitación ponen de manifiesto que sin una revisión de la imagen del ser humano sobre la que se han construido tanto el modelo social, como la teoría tradicional de los derechos, las personas con discapacidad intelectual y psicosocial continuarán siendo excluidas.

Por otro lado, no se tiene en cuenta la condición individual de las personas excluidas, tampoco es posible comprender y valorar las diferencias tal y como exige el objetivo de la igualdad inclusiva, que el Comité sobre los Derechos de las Personas con Discapacidad deriva de la Convención ( $\underline{\mathrm{CRPD} / \mathrm{C} / \mathrm{GC} / 6}, \S 11)$ y que la universalidad de los derechos y exige (Palacios e Iglesias, 2020). 


\section{Bibliografía}

ABBERLEY, P.; "The Concept of Oppression and the Development of a Social Theory of Disability", Disability, Handicap and Society, 2, 1, 1987, 5-19.

ASÍS ROIG, R.; Sobre discapacidad y derechos, Madrid, Dykinson, 2013.

BARNES, C.; Disabled people in Britain and discrimination, London, Hurst and Co., 1991.

BARNES, C.; “The Social Model of Disability. Valuable or irrelevant?”, in N. Watson, A. Roulstone and C. Thomas (Eds.), The Routledge Hadbook of Disability Studies, London, Routledge, 2012, pp. 12-29.

BARNES, C., MERCER, G., and SHAKESPEARE, T.; Exploring Disability. A Sociological Introduction, Cambridge, Polity Press, 1999.

BARNES, M. and SHARDLOW, P.; "Identity Crisis: Mental Health User Groups and the 'Problem' of Identity", in C. Barnes and G. Mercer (Eds.) Exploring the Divide, Leeds, The Disability Press, 1996, pp. 114-134.

BARRANCO AVILÉS, M.C.; "Derechos humanos y vulnerabilidad. Los ejemplos del sexismo y el edadismo", en M.C. Barranco Avilés y C. Churruca Muguruza (Eds.), Vulnerabilidad y protección de los derechos humanos, Valencia, Tirant lo Blanch, 2014, pp. 17-44.

BARRANCO AVILÉS, M.C.; "Democracia, sufragio universal y discapacidad", IgualdaES, 1, 2019, 187-203. Disponible en http://www.cepc.gob.es/publicaciones/revistas/revistaselectronicas?IDR=16\&IDN=1424\&IDA=38809 (último acceso 54-21).

BERNARDINI, M.G.; Disabilità, giustizia, diritto. Itinerari tra filosofia del diritto e Disability Studies, Torino, Giappichelli, 2016.

BERNARDINI, M. G. E GIOLO, O. (Eds.), Le teorie critiche del diritto, Pisa, Pacini, 2017.

BORSAY, A.; "Personal, Trouble or Public Issue?", in L. Burton and M. Oliver (Eds.), Disability Studies: Past, Present and Future, Leeds, The Disability Press, 1997, pp. 115-137.

CAMPOY CERVERA, I.; "Sobre el derecho a una educación inclusiva de calidad", en C. Guilarte y J.J. García Medina (Eds.), Estudios y comentarios jurisprudenciales sobre discapacidad, Pamplona, Aranzadi, 2016, pp. 523-533.

CARLSON, L.; "Philosophers of Intellectual Disability: A Taxonomy", Methaphilosophy, 40, 3-4, 2009, pp. 552-556.

CORKER, M. and FRENCH, S.; "Reclaiming discourse in disability studies", en M. Corker and S. French (Eds.), Disability Discourse, Buckingham, Open University Press, 1999, pp. 1-12.

CORKER, M. and SHAKESPEARE, T. (Eds.); Disability/Postmodernity. Embodying Disability Theory, London, Continuun, 2002.

CUENCA GÓMEZ, P.; "La capacidad jurídica de las personas con Discapacidad: el art. 12 de la Convención de la ONU y su Impacto en el Ordenamiento jurídico español”, Derechos y Libertades, 24, 2011, pp. 221-257.

CUENCA GÓMEZ, P.; "Sobre la inclusión de la discapacidad en la teoría de los derechos humanos", Revista de Estudios Políticos, 158, 2012, pp. 103-137.

CUENCA GÓMEZ, P.; "El derecho al voto de las personas con discapacidad intelectual y psicosocial. La adaptación de la legislación electoral española la Convención Internacional de los Derechos de las Personas con Discapacidad"; Derechos y Libertades, 38, 2018, pp. 171-203.

FINKELSTEIN, V.; Attitudes and disabled people: Issues for Discussion, New York, World Rehabilitation Fund, 1980 Disponible en https://disability-studies.leeds.ac.uk/wp-content/uploads/sites/40/library/finkelstein-attitudes.pdf (último acceso 5-4-21).

FINKELSTEIN, V. ; “To deny or not to deny disability", in Brechin, A., et al. (eds.), Handicap in a Social World, Sevenoaks, Hodder and Stoughton, 1981.

FINKELSTEIN, V.; “The 'Social Model of Disability' and the Disability Movement”, Marzo 2007, disponibile in https://disability-studies.leeds.ac.uk/wp-content/uploads/sites/40/library/finkelstein-The-Social-Model-of-Disability-andthe-Disability-Movement.pdf (ultimo accesso: 5-4-2021). 
FREEMAN, M. A.; "Measuring Equality: a Comparative Perspective on Women's Legal Capacity and Constitutional Rights in Five Commonwealth Countries", Berkeley Women's Law Journal, 5, 1989-1990, pp.110-138.

HUGHES, Z.; "Intellectual disability: caring and role reversal”, The Irish Social Worker, marzo 2016, 66-70.

INE ; El salario de las personas con discapacidad. Año 2018 (29-9-2020), http://www.ine.es/dyngs/INEbase/es/operacion.htm?c=Estadistica_C\&cid=1254736176911\&menu=ultiDatos\&idp=125473 $\underline{5976596}$ (última consulta 5-4-2021).

KANT, I. ; Teoría y Práctica, trad. R. Rodríguez Aramayo, Madrid, Tecnos, 2006.

KITTAY, E. F.; "Equality, Dignity and Disability", in M.A. Lyons and F. Waldron (Eds.), Perspectives on Equality: The Second Seamus Heaney Lectures, Dublin, The Liffey Press, 2005, pp. 93-119.

MCRUER, R., Teoría Crip. Signos culturales de lo queer y la discapacidad, trad. J. Sáez, Madrid, Kaotica, 2021.

MEEKOSHA, H and SHUTTLEWORTH, R., "What's so 'critical' about critical disability studies?", Australian Journal of Human Rights, Vol. 15 (1), 2009, pp. 47-75.

MOSCOSO PÉREZ, M., y ARNAU RIPOLLÉS, S., "Lo Queer y lo Crip, como formas de re-apropiación de la dignidad disidente. Una conversación con Rober McRuer”, Dilemata, 8, pp. 137-144, 2016.

OLIVER, M.; The politics of disablement, Basignstoke, Macmillan, 1990.

OLIVER, M. and BARNES, C; "Disability politics and the disability movement in Britain: Where did it all go wrong?", June 2006, disponibile en https:/disability-studies.leeds.ac.uk/wp-content/uploads/sites/40/library/Barnes-Coalition-disabilitypolitics-paper.pdf (ultimo accesso: 5-4-2021).

PALACIOS, A.; El modelo social de discapacidad: orígenes, caracterización y plasmación en la Convención Internacional sobre los Derechos de las Personas con Discapacidad, Madrid, CINCA, 2008.

PALACIOS, A. E IGLESIAS, M.G.; "La discapacidad y los valores que sustentan a los derechos humanos", en A . Palacios, S. E. Fernández y M.G. Iglesias, Situaciones de discapacidad y derechos humanos, Buenos Aires, La Ley, 2020, pp. 91-132.

PODER JUDICIAL DE COSTA RICA; Protocolo de Atención para el Efectivo Acceso a la Justicia de Personas con Discapacidad Psicosocial, Eurosocial, 2013, disponibile su http://sia.eurosocial-ii.eu/files/docs/1396253595Protocolo_Costa_Rica_Acceso_Justicia_personas_discapacidad.pdf, (último accesso: 5-4-2021).

QUINN, G. and ARSTEIN-KERSLAKE, A.; "Restoring the 'human' in 'human rights': personhood and doctrinal innovation in the UN Disability Convention", in Gearty, C. and Douzinas, C. (Eds.) Human Rights Law, Cambridge, Cambridge University Press, 2012, pp. 36-55.

QUINN, G. and DEGENER, T. (et al.); The current use and future pontetial of United Nations human rights instrument in the context of disability, New York and Geneva, United Nations, 2002.Reindal, S. M. (1999). Independence, Dependence, Interdependence: some reflections on the subject and personal autonomy. Disability and Society, 14, 3, 353-367.

RYAN, J. and THOMAS, F.; The Politics of Mental Handicap, Harmondsworth, Penguin Books, 1980.

SHAKESPEARE, T. and WATSON, N; "The social model of disability: an outdated ideology?", Research in Social Science and Disability, 2, 2002, pp. 9-28.

SHAPIRO, J.; No Pity. People with Disabilities Forging a New Civil Rights Movement, New York, Three Rivers, 1994.

SIEBERS, T.; Disability Theory, Michigan (U.S.), University of Michigan Press, 2008.

SIERRA, M. L.; Mujeres con discapacidad: sobre la discriminación y opresión interseccional, Madrid, Dykinson, 2017.

SIMPLICAN, S.C.; "Making disability public in deliberative democracy", Contemporary Political Theory. 11, 2, 2012, pp. 211-228.

SIMPLICAN, S.C.; The Capacity Contract. Intellectual Disability and the Question of Citizenship, Minneapolis and London, University of Minnesota Press. 2015.

TROILO, S.; Tutti per uno o uno contro tutti? Il diritto all 'istruzione e all 'integrazione scolastica dei disabili nella crisi dello stato sociale, Torino, Giuffrè, 2012, 
TRONTO, J. C.; "Universalistic Morality and Moral Sentiments", Moral Boundaries. A Political Argument for an Ethic of Care, New York, Routledge, 1993.

UNICEF, Evitar una generación perdida a causa de la COVID-19. New York, UNICEF, 2020. Disponible en https://www.unicef.es/sites/unicef.es/files/comunicacion/Evitar_una_generacion_perdida_a_causa_de_la_COVID.pdf (último acceso 5-4-21).

YOUNG, I. M.; Justice and the Politics of Difference, New Jersey, Princeton University Press, 2011. 